\title{
BASINS FOR GENERAL NONLINEAR HÉNON ATTRACTING SETS
}

\author{
SAM PATTERSON AND CLARK ROBINSON
}

(Communicated by Kenneth R. Meyer)

\begin{abstract}
When a new homoclinic intersection is created for a dissipative diffeomorphism in dimension two, there results a cascade of sinks. We show that immediately after one of these sinks $q_{n}$ is formed, its basin boundary is made up of the stable manifold of the saddle periodic point $p_{n}$ formed at the same time. After this sink undergoes a period doubling, there still remains a trapping region with an attracting set inside. In fact, we show that until this saddle periodic point $p_{n}$ has its own homoclinic bifurcation, there is an attracting set whose boundary is made up of the stable manifold of $p_{n}$. By picking a rectangle $B_{n}$ carefully, the one-parameter family of maps $f_{t^{n}}$ creates these sinks and attracting sets by pulling the image $f_{t^{n}}\left(B_{n}\right)$ across $B_{n}$ and eventually forming a horseshoe in $B_{n}$. The maps, $f_{t^{n}}$ on $B_{n}$, are well approximated for large $n$ by quadratic maps equivalent to the Hénon map. We prove our results for general nonlinear Hénon maps which include not only the quadratic maps but also other nonlinear maps which also create horseshoes, including those arising from homoclinic tangencies.
\end{abstract}

1. Statement of results. When a new homoclinic intersection is created for a dissipative diffeomorphism in dimension two, there results a cascade of sinks $[6,13$, 14]. The problem, which we addressed in our recent paper [12] and in the theorem below, is to determine something of the shape and size of the basins of these periodic sinks created in these cascades. We want to prove these results for general classes of functions from dynamical assumptions, in particular from assumptions on the stable and unstable manifolds of the saddle periodic point. The proof of the existence of these sinks (following Newhouse [9]) shows that there exist rectangles $B_{n}$ for $n \geq N$ such that as $t$ varies $F_{t}^{n}\left(B_{n}\right)$ is pulled across $B_{n}$, forming a horseshoe. The question then becomes the following: when a dissipative map $f_{t}\left(=F_{t}^{n}\right)$ creates a horseshoe in $B\left(=B_{n}\right)$, what is the basin of the sink formed by the saddle node bifurcation?

We have focused on the case when $f_{t}$ is orientation preserving and decreases area in $B$. In $\S 2$, we state the explicit assumption, (A1)-(A7), on $f_{t}$ and $B$ which are sufficient to show that $f_{t}$ forms a nonlinear horseshoe and then to determine the basin of the attracting set. The simplest such map is quadratic and is really the Hénon map written in different coordinates and with different parameters:

$$
f_{t}(x, y)=\left(\lambda y, t-\mu x+y+\Delta y-\lambda^{2} y^{2}\right)
$$

where $0<\mu<1<\lambda$ and $\Delta=\lambda \mu<1$. (Here $\Delta$ is the factor by which area is changed.) The proof in [4] shows how to pick the rectangle $B$. Note that a simple

Received by the editors November 2, 1987.

1980 Mathematics Subject Classification (1985 Revision). Primary 58F 15, 58F 13.

Key words and phrases. Basins, sinks, homoclinic tangencies, creation of horseshoes.

Partially supported by a grant from the National Science Foundation. 

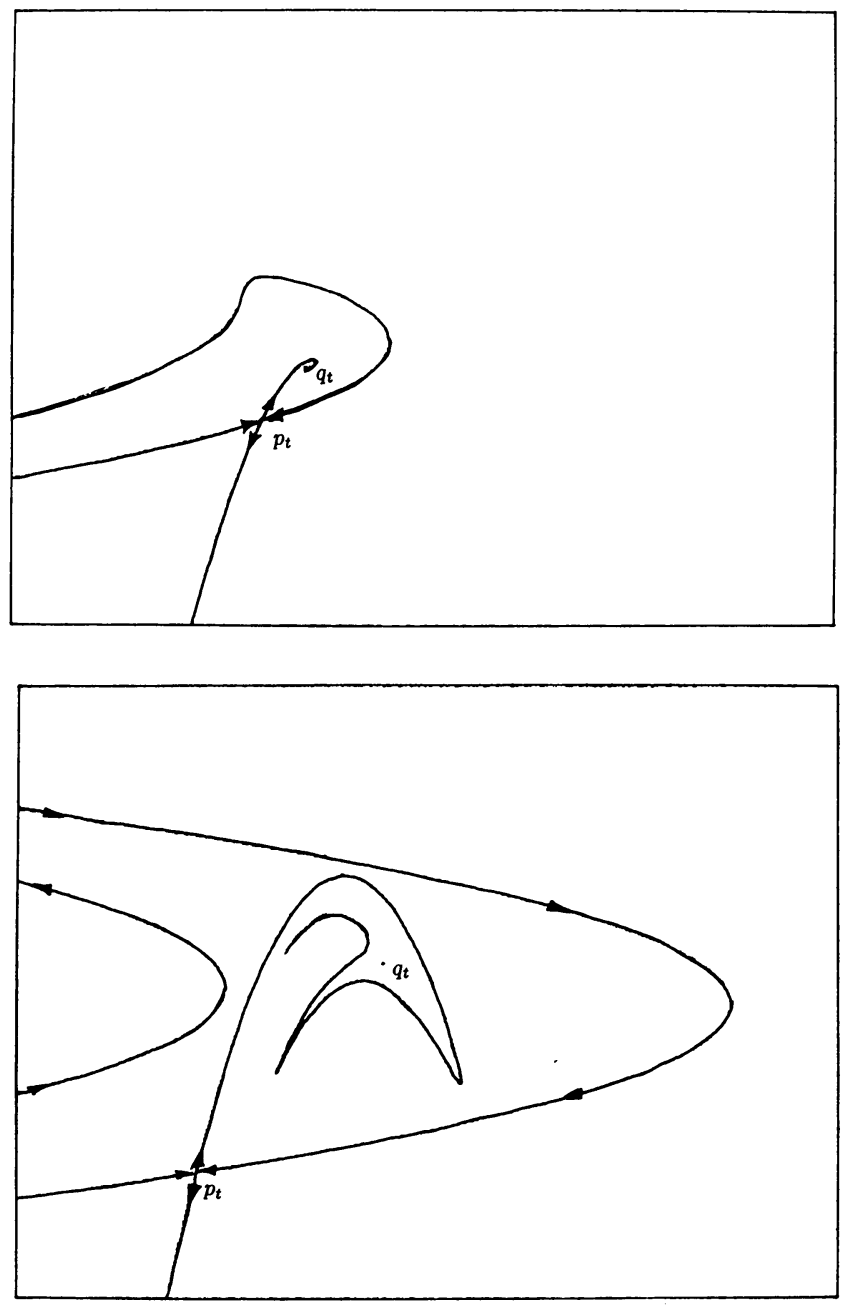

FIGURE 1. $W^{s}\left(p_{t}, F_{t}\right)$ and $W^{u}\left(p_{t}, F_{t}\right)$ for $(*)$ with

$$
\mu=\frac{1}{4}, \lambda=2 \text {, and } t-t_{0}=0.02 \text { and } 0.3
$$

change of coordinates is needed to change our form of the map into that given in the reference.

In [12], we determined the local basin of attraction of the sink immediately after the saddle node bifurcation, i.e., the result is true as a perturbation of the saddle node situation. Let $p_{t}$ be the saddle, $q_{t}$ the sink, and $B_{t}^{-}=f_{t}^{-1}(B)$. We proved that immediately after the saddle node bifurcation, the local basin of $q_{t}$ (given by the component of $W^{s}\left(q_{t}, f_{t}\right)$ in $\left.B \cup B_{t}^{-}\right)$is the same as the component of $B \cup B_{t}^{-}-W^{s}\left(p_{t}, f_{t}\right)$ containing $q_{t}$. Thus the stable manifold of the saddle forms the basin boundary for the sink.

Since we want to follow this basin for parameter values where $q_{t}$ has period doubled and ceased to be a sink, we need to look at the attracting set in this region. By an attracting set $A$ for a map $f$, we mean there is a trapping region $V$ such that $f($ closure $V) \subset$ interior $V$ and $A=\bigcap_{n \geq 0} f^{n}(V)$. In this paper we prove the following result which was conjectured in our previous paper. It states that 
prior to the first homoclinic bifurcation of $p_{t}$, there is an attracting set $A_{t}$ whose basin contains this same component of $B \cup B_{t}^{-}-W^{s}\left(p_{t}, f_{t}\right)$.

THEOREM. Assume $\left\{f_{t}\right\}$ and $B$ satisfy $(*)$ or the assumptions (A1)-(A7) given in $\S 2$. In particular, $f_{t}$ is orientation preserving and decreases area. Further assume that $t>t_{0}$, where $t_{0}$ is the saddle node bifurcation value, and that $p_{t}$ has not gone through a homoclinic bifurcation, i.e., $W^{s}\left(p_{t}, f_{t}\right) \cap W^{u}\left(p_{t}, f_{t}\right)-\left\{p_{t}\right\}=\varnothing$. Then there is an attracting set $A_{t} \subset B \cup B_{t}^{-}$, where $B_{t}^{-}=f_{t}^{-1}(B)$, such that the component of the basin of attraction of $A_{t}$ in $B \cup B_{t}^{-}$is bounded by $W^{s}\left(p_{t}, f_{t}\right)$ and a line segment in the boundary of $B$.

The proof of this theorem is contained in $\S \S 3$ and 4. The main part of the proof involves determining the location and shape of the stable and unstable manifolds, $W^{s}\left(p_{t}, f_{t}\right)$ and $W^{u}\left(p_{t}, f_{t}\right)$, of the saddle fixed point $p_{t}$. (See Lemma 3.2 and its proof in $\S 4$.) Computer simulation for the Hénon family, $(*)$, easily determines the shape of these curves even more definitely than we were able to prove. In addition to determining these positions rigorously for this particular family, we have determined quite general assumptions on a general nonlinear family of maps, (A1)-(A7), which are sufficient to obtain the same conclusion. Compare these results to those for area preserving maps given in Devaney, [5].

Several other people have studied the basin boundary of the attracting set after the first homoclinic bifurcation (as opposed to before, as done in this paper). Let $t_{h}^{*}$ be the value of the parameter at the first homoclinic bifu.cation of $p_{t}$, and $T^{*}$ the parameter value with the last homoclinic bifurcation of $p_{t}$. In the range $t_{h}^{*}<z<T^{*}$, the basin boundary ceases to be made up of $W^{s}\left(p_{t}, f_{t}\right)$, but rather the stable manifold of a higher periodic point. See $[\mathbf{1}, \mathbf{2}, \mathbf{7}$, and 8]. Further work remains to completely understand the dynamics during the formation of the horseshoe.

As stated at the beginning of this paper, a cascade of sinks is created whenever a dissipative saddle point creates a new homoclinic intersection at a parameter value $t_{0}$. In fact, S. Newhouse proved in [9] that with the nondegeneracy assumption that there are many parameter values near $t_{0}$ for which there are infinitely many periodic sinks (for the same parameter value). Recently at the International Congress of Mathematicians in Berkeley, California, F. Takens questioned the proof of the theorem and felt there was a gap in showing there was a horseshoe with large stable thickness. Since then, both S. Newhouse and the second author of this paper have clarifications of the original proof which show that it is valid.

More recently, G. Davis has shown that the same result is true (that there are infinitely many sinks) with the weaker assumption that the family has a homoclinic bifurcation with finite order contact. In particular, this occurs if the family is real analytic and the stable and unstable manifolds do not coincide for one parameter value. See [3].

2. Assumption on general nonlinear Hénon family. In this section, we give the assumptions on a nonlinear family that are sufficient to prove the theorem. They are similar to those in [12]. The first six assumptions are quite general and merely mean that a horseshoe is formed in a topological sense: there is a rectangle $B$ whose image, $f_{t}(B)$, is shaped like a horseshoe and is pulled across $B$ as $t$ varies. The seventh and last assumption gives conditions on the first and 
second derivatives of the coordinate functions which are sufficient to show that these derivatives determine the shape of $f_{t}(B)$. In particular, (A7ii) and (A7iii) imply $\left\{\left(f_{t}\right)_{2}(x, y)-y=0\right\}$ and $\left\{\left(f_{t}^{-1}\right)_{1}(x, y)-x=0\right\}$ are convex to the left and downward respectively. See Lemma 4.1. Finally (A7iv) insures the second derivative condition at the saddle node. We express these specific assumptions in terms of a choice of local coordinates but they clearly have intrinsic geometric meaning.

Let $B=\left[x_{1}, x_{2}\right] \times\left[y, y_{2}\right]$ and let $\left\{f_{t}\right\}$ be a one-parameter family of maps for $t_{-1} \leq t \leq t_{1}$.

(A1) (i) For each $t$ in $\left[t_{-1}, t_{1}\right]$, both $f_{t}$ and $f_{t}^{-1}$ are defined on $B$ so that the domain of $f_{t}$ includes $B \cup B_{t}^{-}$where $B_{t}^{-}=f_{t}^{-1}(B)$. (ii) For each $t$ in $\left[t_{-1}, t_{1}\right], f_{t}$ is $C^{2}$. (iii) $f_{t}$ varies continuously in the $C^{2}$ topology as $t$ varies.

(A2) The family preserves orientation and decreases area in $B \cup B_{t}^{-}$for each $t$, i.e., $0<\operatorname{det} D f_{t}(x, y)<1$ for all $(x, y)$ in $B \cup B_{t}^{-}$and $t_{-1} \leq t \leq t_{1}$.

(A3) For $t=t_{-1}, f_{t}(B) \cap B=\varnothing$.

(A4) Let $t=t_{1}$ and fix any $x_{0}$ in $\left[x_{1}, x_{2}\right]$. As $y$ increases from $y_{1}$ to $y_{2}, f_{t_{1}}\left(x_{0}, y\right)$ starts outside $B$ and below $B,\left(f_{t_{1}}\right)_{2}\left(x_{0}, y_{1}\right)<y_{1}$; then $f_{t_{1}}\left(x_{0}, y\right)$ crosses $B$ and exits the top of $B,\left(f_{t_{1}}\right)_{2}\left(x_{0}, y\right)>y_{2}$; and $f_{t_{1}}\left(x_{0}, y\right)$ reenters and crosses $B$ again and finally exits the bottom of $B,\left(f_{t_{1}}\right)_{2}\left(x_{0}, y_{2}\right)<y_{1}$.

(A5) $f_{t}(B)$ never intersects the sides of $B$ for $t_{-1} \leq t \leq t_{1}, f_{t}(B) \cap\left(\left\{x_{j}\right\} \times\right.$ $\left.\left[y_{1}, y_{2}\right]\right)=\varnothing$ for $j=1,2$. Also,the first coordinate of $f_{t}^{-1}\left(x_{j}, y\right)$ is less than $x_{1}$ for $j=1,2$ and $t_{-1} \leq t \leq t_{1},\left(f_{t}^{-1}\right)_{1}\left(x_{j}, y\right)<x_{1}$.

(A6) For $j=1,2$ and $t_{-1} \leq t \leq t_{1},\left(f_{t}\right)_{2}\left(x, y_{j}\right)<y_{1}$. It follows that $f_{t}^{-1}(B)$ does not intersect the top or bottom of $B$. Further assume $y_{1}<\left(f_{t}^{-1}\right)_{2}(x, y)<y_{2}$ for all $(x, y)$ in $B \cup B_{t}^{-}$and $t_{-1} \leq t \leq t_{1}$.

(A7) Let $D f_{t}(x, y)=\left(\begin{array}{ll}a & b \\ c & d\end{array}\right)$ where $a, b, c, d$ depend on $(x, y)$ and $t$. Assume, for $t_{-1} \leq t \leq t_{1}$ and $(x, y)$ in $B \cup B_{t}^{-}$, that

(i) $b c<0$ and $|a|<\Delta<\operatorname{det} D F_{t}$,

(ii) $D^{2}\left(f_{t}\right)_{2}(v, v)<0$, where the column vector $v=(d-1,-c)^{T}$ is acted on by the bilinear map formed by the second derivative of the second coordinate function evaluated at $(x, y)$, i.e., dropping the subscript to indicate the dependence of the parameter $t$,

$$
(-c)^{2}\left(\partial^{2} f_{2} / \partial y^{2}\right)+2(-c)(d-1)\left(\partial^{2} f_{2} / \partial y \partial x\right)+(d-1)^{2}\left(\partial^{2} f_{2} / \partial x^{2}\right)<0
$$

(iii) $D^{2}\left(f_{t}\right)_{2}(w, w)+(d /-b) D^{2}\left(f_{t}\right)_{1}(w, w)<0$ for $w=(b, 1-a)^{T}$ and

(iv) $D^{2}\left(f_{t}\right)_{2}(w, w)+(a-\Delta / b) D^{2}\left(f_{t}\right)_{1}(w, w)<0$ for $w=(b, 1-a)^{T}$.

3. Proof of the theorem. To simplify notation, we often drop the subscript denoting the parameter $t$ and write $f$ for the function $f_{t}$, and $p$ for the saddle fixed point $p_{t}$ of $f_{t}$. We also write $W^{s}(p)$ for the stable manifold of this fixed point, i.e., for $W^{s}\left(p_{t}, f_{t}\right)$, and $W^{u}(p)$ for $W^{u}\left(p_{t}, f_{t}\right)$.

For definiteness of the discussion in the proof, we make certain choices for signs of the partial derivatives of the coordinate functions $f_{1}$ and $f_{2}$ (or $f_{t 1}$ and $f_{t 2}$ ):

$$
b=\frac{\partial f_{1}}{\partial y}>0, \quad c=\frac{\partial f_{2}}{\partial x}<0, \quad \text { and } \quad \frac{\partial^{2} f_{2}}{\partial y^{2}}<0,
$$

where the values of the quantities depend on $x, y$, and $t$.

Using (A7iv), it is shown in [12] that there is a parameter value $t_{0}$ at which a saddle node bifurcation takes place. Throughout the proof, we only consider 
parameter values $t$ which are larger than this bifurcation value $t_{0}$. We could proceed without assumption (A7iv) and then only consider parameter values $t$ for which $f$ has a saddle fixed point in $B$.

LEMMA 3.1. For $t>t_{0}$ there are exactly two fixed points of $f_{t}$ inside $B$. The lower left fixed point $p_{t}$ is an untwisted saddle with index -1 and the upper right fixed point $q_{t}$ has index +1 and is either a sink or a twisted saddle point (i.e., has negative eigenvalues).

For the proof see $\S 4$.

The next lemma states that $W^{s}(p)$ is outside of $W^{u}(p)$ and extends to the left end of $B$. Some notation is useful to make this description. Let $R^{s}$ be the right branch of $W^{s}(p)$ and $L^{s}$ the left branch (using the directions that the branches, $\operatorname{comp}\left(W^{s}(p)-p\right)$, leave $\left.p\right)$. Let $T^{u}$ be the top branch of $W^{u}(p)$.

LEMMA 3.2. (a) The left branch of the stable manifold of $p, L^{s} \subset W^{s}(p)$, extends to the left end of $B$ without crossing the bottom of $B$.

(b) The right branch of the stable manifold of $p, R^{s} \subset W^{s}(p)$, goes outside the top branch of the unstable manifold, $T^{u} \subset W^{u}(p)$, and extends to the left end of $B$ without crossing the top or bottom of $B$. (It is contained in $B \cup f^{-1}(B)$ up to the points where it crosses this left end.)

(c) In particular, by picking the points of $R^{s}$ and $L^{s}$ closest together along $x=x_{1}$ and connecting them with a line segment in $x=x_{1}$, there results a closed curve $\Gamma$ surrounding an open disk $D_{1}$ with $f\left(D_{1}\right)=D_{1}$.

Part (b) is the key step. The idea is that since $W^{s}(p) \cap W^{u}(p)=\{p\}$ either $R^{s}$ goes outside $T^{u}$ or inside. Because of the area decreasing assumption, we show that $R^{s}$ must go outside. The complete proof requires a very careful argument and is given in $\S 4$.

The only reason that $D_{1}$ is not the desired trapping region is that closure $f\left(D_{1}\right)$ is not contained inside interior $D_{1}(f(\Gamma) \cap \Gamma \neq \varnothing)$. To obtain a trapping region $D \subset D_{1}$, we trim off the edges as given in the following lemma. This lemma completes the proof of the theorem.

LEMMA 3.3. There is a trapping region $D \subset D_{1}$ with $f($ closure $D) \subset$ interior $(D)$ and defining $\Lambda=\bigcap_{j>0} f^{j}(D)$ then (i) all points of $D_{1}-D$ are wandering with forward orbit entering $\bar{D}$, so (ii) all points of $D_{1}$ are in the basin of $\Lambda$. Thus the basin boundary for the attracting set contains that part of $\partial D_{1}$ lying in $R^{s} \cup L^{s}$.

PROOF. The trapping region $D$ is constructed using the $\lambda$-lemma, [11]. Take $L$ a line segment transverse to $T^{u}$ and $L \subset D_{1}$. Let $L_{N}=f^{-N}(L)$. This is a curve near $W^{s}(p)$ in the $C^{1}$ topology. Near $p, f\left(L_{N}\right)$ is on the side of $L_{N}$ away from $p$ and toward the interior of $D_{1}$. Thus by taking $N$ large, $L_{N}$ extends along the boundary of $D_{1}$ made up of $W^{s}(p)$ and $f\left(L_{N}\right)$ is on the side of $L_{N}$ away from the boundary of $D_{1}$. Let $D$ be the open set with boundary $L_{N}$ and part of $x=x_{1}$. Then $f$ (closure $D) \subset$ interior $D$. (Note that $f$ (boundary $D$ ) does not cross the boundary of $D$.)

By the $\lambda$-lemma the points in $D_{1}-D$ are trapped between iterates $f^{-j}\left(L_{N}\right)$ and $f^{-j-1}\left(L_{N}\right)$ for some $j \geq 0$ and most enter $D$ under forward iteration. 
4. Proof of Lemmas 3.1 and 3.2. The fixed points and general shape and location of $W^{s}(p)$ and $W^{n}(p)$ are found by first determining the curves where one of the coordinates is fixed by $f$ or $f^{-1}$. For $z=(x, y)$, let

$$
\begin{aligned}
\delta_{x}(z ; t) & =\left(f_{t}\right)_{1}(z)-x, & & \delta_{y}(z ; t)=\left(f_{t}\right)_{2}(z)-y, \\
\delta_{x}^{-}(z ; t) & =\left(f_{t}^{-1}\right)_{1}(z)-x, & & \delta_{y}^{-}(z ; t)=\left(f_{t}^{-1}\right)_{2}(z)-y,
\end{aligned}
$$

where $\left(f_{t}\right)_{j}$ is the $j$ th coordinate function of $f_{t}$. Lemma 4.1 discusses the shape of $\left\{\delta_{x}=0\right\},\left\{\delta_{y}=0\right\},\left\{\delta_{x}^{-}=0\right\}$, and $\left\{\delta_{y}^{-}=0\right\}$. Let $E_{t}^{s}$ and $E_{t}^{u}$ be the stable and unstable lines at the fixed point $p_{t}$. It follows from the assumptions that the slopes of both $E_{t}^{s}$ and $E_{t}^{u}$ are positive and that of $E_{t}^{s}$ is less than $E_{t}^{u}$.

LEMMA 4.1. (a) The curve $\left\{\delta_{y}=0\right\}$ is given by a graph $x=h(y)$ with $h^{\prime \prime}(y)<$ 0 and with $h(y)$ having one maximum. Further, the region $\left\{\delta_{y} \geq 0\right\}$ is the convex set to the left of $\left\{\delta_{y}=0\right\}$.

(b) The curve $\left\{\delta_{x}^{-}=0\right\}$ is given by a graph $y=g(x)$ with $g^{\prime \prime}(x)<0$ and with $g(x)$ having one maximum. The region $\left\{\delta_{x}^{-} \geq 0\right\}$ is the convex set below the curve $\left\{\delta_{x}^{-}=0\right\}$.

(c) The two curves $\left\{\delta_{x}=0\right\}$ and $\left\{\delta_{y}^{-}=0\right\}$ each have strictly positive slope.

This lemma follows from assumption (A7) (or is a direct calculation for the quadratic map (*)) and is the only place this assumption is used. Part (a) follows directly from (A7ii) using the implicit function theorem. Similarly, (b) follows from (A7iii), but here a calculation is needed to show that the necessary conditions on the second partial derivatives of $f^{-1}$ follow from the ones given on $f$. Part (c) follows from (A7i) using the fact that $\Delta-a$ and $1-a$ are positive. The details are found in $[\mathbf{1 2}]$.

PROOF OF LEMMA 3.1. The fixed points are exactly the points of intersection of $\left\{\delta_{y}=0\right\}$ and $\left\{\delta_{x}=0\right\}$. When the saddle node bifurcation takes place for $t=t_{0}$, the lower left fixed point formed is an untwisted saddle fixed point (both eigenvalues are positive). As $t$ increases, there remains a fixed point, $p$, at the lowest level along $\left\{\delta_{x}=0\right\}$. By following the signs of the charge of the $x$ and $y$ components, it follows that this point has index -1 for all $t>t_{0}$.

For $t>t_{0},\left\{\delta_{x}=0\right\}$ crosses to the left side of $\left\{\delta_{y}=0\right\}$ at $p$, so it must cross again. Thus the number of fixed points is at least two.

If $\left\{\delta_{x}=0\right\}$ crosses $\left\{\delta_{y}=0\right\}$ on the top branch where $d y / d x<0$, then since the slope of $\left\{\delta_{x}=0\right\}$ is everywhere positive it cannot cross again. Thus, in this case, there are exactly two fixed points.

If $\left\{\delta_{x}=0\right\}$ crosses $\left\{\delta_{y}=0\right\}$ on the bottom branch where $d y / d x>0$, then more care must be taken. If it does not cross the bottom branch again, then it clears the end of $\left\{\delta_{y}=0\right\}$ so there are exactly two fixed points.

To complete the proof, we must show that it is impossible for $\left\{\delta_{x}=0\right\}$ to cross the bottom branch of $\left\{\delta_{y}=0\right\}$ three times. Let these points be $p, q_{1}$, and $q_{2}$ given in the order of increasing $x$. At $q_{1},\left\{\delta_{x}=0\right\}$ crosses $\left\{\delta_{y}=0\right\}$ from top to bottom so $0<$ slope $\left\{\delta_{x}=0\right\}_{q_{1}} \leq$ slope $\left\{\delta_{y}=0\right\}_{q_{1}}$. Similarly, $0<$ slope $\left\{\delta_{y}=0\right\}_{q_{1}} \leq$ slope $\left\{\delta_{x}=0\right\}_{q_{2}}$. These are all fixed points so $\left\{\delta_{x}^{-}=0\right\}$ goes through these points. This contradicts the fact that $d^{2} y / d x^{2}<0$ on $\left\{\delta_{x}^{-}=0\right\}$ and $d^{2} y / d x^{2}>0$ on $\left\{\delta_{y}=0\right\}$.

The sum of the indices must remain 0 , and $p$ has index -1 so $q$ has index +1 . 
PROOF OF LEMMA 3.2. To prove this lemma, we must show in the next two lemmas that $R^{s}$, the right branch of $W^{s}(p)$, crosses $\left\{\delta_{x}=0\right\}$ above $q$. We give this part of $\left\{\delta_{x}=0\right\}$ above $q$ the name $J$. Similarly, we must show that $T^{u}$, the top branch of $W^{u}(p)$, either goes to $q$ or crosses $J$.

We also need to distinguish between $R^{s}$ going directly from $p$ to a point $z$ of $R^{s} \cap J$ and $R^{s}$ winding around $q$ before reaching $z$. Therefore for $z \in J \cap R^{s}$ we assign the winding number, $N(z)=N^{s}(z)$, to be the number of times $R^{s}$ goes around $q$ before reaching $z$. Let $r$ be the first point of intersection of $R^{s}$ with $J$ and set $N(r)=0$. For a further point $z \in R^{s} \cap J$ connect $z$ to $r$ along $J$ and take the part of $R^{s}$ between the two points. Then $N(z)$ is the number of times this curve goes around $q$. (Remember that $J$ is only the part of $\left\{\delta_{x}=0\right\}$ above $q$.) Similarly, for $z \in T^{u} \cap J$, let $N(z)=N^{u}(z)$ be the winding number of $T^{u}$ around $q$.

LEMMA 4.2. (a) $R^{s}$ extends up and crosses $J \subset\left\{\delta_{x}=0\right\}$ at a finite distance along $R^{s}$ from $p$. In fact, if $D^{s}$ is a fundamental domain in $R^{s}$, then there is an integer $I>0$ such that for any $w \in D^{s}$ there is an integer $i$ such that $0<i<I$ and $f^{-i}(w)$ is not in $\left\{\delta_{x}=0\right\}$.

(b) If $z \in J \cap R^{s}$ with $N(z)=0$, then $O^{+}(z) \subset\left\{\delta_{x}<0\right\}$.

PROOF. (a) The-slope of $R^{s}$ at $p$ is less than that of $\left\{\delta_{x}=0\right\}$. Thus $R^{s}$ enters the region $\left\{\delta_{y}=0\right\} \cap\left\{\delta_{x}<0\right\} \cap\left\{\delta_{y}^{-}>0\right\} \cap\left\{\delta_{x}^{-}>0\right\}$. It is also above $y=y_{0}$ where $p=\left(x_{0}, y_{0}\right)$. Since $\delta_{y}^{-}>0$ on $\left\{\left(x, y_{0}\right): x>x_{0}\right\}, R^{s}$ does not cross this ray before leaving $\left\{\delta_{x}<0\right\}$. Thus it does not cross $f^{-1}\left\{\left(x, y_{0}\right): x>x_{0}\right\}$. Let $H$ be the region bounded by $f^{-1}\left\{\left(x, y_{0}\right): x>x_{0}\right\},\left\{\delta_{y}=0\right\}$ and $\left\{\delta_{x}=0\right\}$. The arc $R^{s}$ enters $H$. It cannot leave across $f^{-1}\left\{\left(x, y_{0}\right)\right\}$.

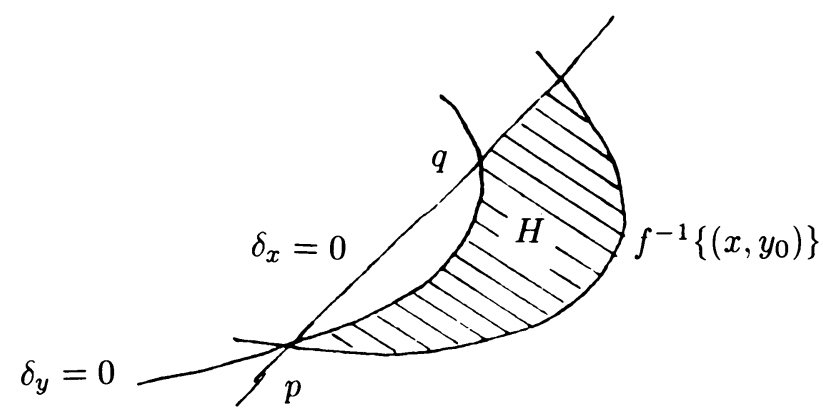

FIGURE 2

Now consider that piece of boundary of $H$ consisting of $\left\{\delta_{y}=0\right\}$ between $p$ and $q$. Let $z$ lie on this segment. We have $z \in\left\{\delta_{y}=0\right\}$ and, from the proof of Lemma 3.1, $z \in\left\{\delta_{x}<0\right\}$ so $f(z)$ is directly to the left of $z$. We see that points "flow" into $H$ across $\left\{\delta_{y}=0\right\}$ between $p$ and $q$ and thus that $R^{s}$ cannot exit $H$ across this boundary. Consequently, $R^{s}$ either stays in $H$ or leaves $H$ across $J$.

Let $\hat{H}=H \cap\left\{\delta_{x}^{-}<0\right\}$. Since $f^{-1}\left\{\delta_{x}^{-}=0\right\}=\left\{\delta_{x}=0\right\}, R^{s}$ leaves $H$ across $\left\{\delta_{x}=0\right\}$ if and only if $R^{s}$ leaves $\hat{H}$ across $\left\{\delta_{x}^{-}=0\right\}$. Let $w \in R^{s} \cap \hat{H}$ and suppose $O^{-}(w) \subset \hat{H}$. Since $\delta_{x}^{-}>0$ in $\hat{H}$ and $\delta_{y}^{-}>0$ in $\hat{H}$ the sequence $O^{-}(w) f$ is monotone in each coordinate and thus must converge to a fixed point which by Lemma 3.1 must be $q$. But $q$ is either a sink, in which case $R^{s} \subset W^{s}(p)$ cannot 
accumulate on $q$, or $q$ is a twisted saddle in which case $O^{-}(w)$ cannot accumulate on $q$ from strictly inside $H$. We conclude that $R^{s}$ leaves $H$ via $J$ and that there exists a positive integer $i$ such that $f^{i}(w) \notin\left\{\delta_{x}<0\right\}$.

Finally let $w$ lie on a fundamental domain $D^{s}$ in $R^{s}$. By compactness of $D^{s}$, there exists $I>0$ such that for each $w \in D^{s}$ there is an integer $i$ such that $0<i \leq I$ and $f^{-i}(w) \notin\left\{\delta_{y}<0\right\}$. This completes the proof of Lemma 4.2(a).

PROOF OF LEMMA 4.2(b). Let $z \in J \cap R^{s}$. If $O^{+}(z)$ leaves $\left\{\delta_{x}<0\right\}$ it must do so below $q$. In this case $O^{+}(z)$ must return to $H$. From the proof of part (a), we know that $O^{+}(z)$ must reenter $H$ across $J$. Thus if $O^{+}(z) \not \subset\left\{\delta_{x}<0\right\}$, then $N(z) \geq 1$.

REMARK. Lemma 4.2 does not quite show that $W^{s}(p) \cap W^{u}(q)=\varnothing$. This fact will follow below from the positions of $W^{s}(p)$ and $W^{u}(p)$ determined in the complete proof of Lemma 3.2.

LEMMA 4.3. Assume $T^{u}$ does not accumulate on $q$ by points whose entire backward orbit lies inside $\left\{\delta_{x}>0\right\}$. Then

(a) if $D^{u}$ is a fundamental domain in $T^{u}$, then there is an $I=I_{t}>0$ such that for any $w \in D^{u}$ there is a $0<i<I$ such that $f^{i}(w) \notin\left\{\delta_{x}>0\right\}$.

(b) If $z \in J \cap T^{u}$ with $N(z)=0$, then $O^{-}(z) \subset\left\{\delta_{x}>0\right\}$.

The proof is similar to that of Lemma 4.2 and is omitted. Combining Lemmas 4.2 and 4.3 , we can prove Lemma 3.2.

PROOF OF LEMMA 3.2(a). $L^{s}$ enters the region where $\left\{\delta_{y}^{-}<0\right\}$ and $\left\{\delta_{x}^{-}<0\right\}$. It cannot cross the bottom of $B$ by assumption $A 4$. Thus it must exit the left end of $B$.

ProOF OF LemMa 3.2(b). By Lemma 4.2, the set $\left\{z \in R^{s} \cap J: N(z)=0\right\}$ is contained in a compact piece $S^{\prime}$ of $R^{s}$. We want to show that $T^{u}$ must go inside $S^{\prime}$ (or directly to $q$ ). Let us assume that $T^{u}$ goes outside $S^{\prime}$ and we will get a contradiction. (We allow $T^{u}$ to have some previous loops crossing $J$ below $S^{\prime} \cap J$ but the last crossing must be above.) To exit outside $S^{\prime}$ it must do so above the highest point of $S^{\prime} \cap J$. Let $S \subset S^{\prime}$ be the part of $R^{s}$ from $p$ to this highest point $z^{\prime}$ of $S^{\prime} \cap J$. Note $N\left(z^{\prime}\right)$ must be zero, because $L^{s}$ blocks $R^{s}$ from returning around $p$ and above $\left\{z \in R^{s} \cap J: N(z)=0\right\}$. Let $U \in T^{u}$. Let $z^{\prime \prime}$ be the closest point to $z^{\prime}$ of $\left\{z \in T^{u} \cap J: N(z)=0\right\}$ that lies above $z^{\prime}$. Let $U$ be the part of $T^{u}$ from $p$ until $z^{\prime \prime}$. Let $C \subset J$ be the connection from the ends of $U$ and $S$ along $J$. Let $\Gamma=\{p\} \cup S \cup U \cup C$. Clearly $\Gamma$ is a simple closed curve. It surrounds $q$. So the region $D$ inside $\Gamma$ contains $q$.

We claim $f(\Gamma)$ does not cross $\Gamma$ and $f(D) \supset D$. We prove this claim in steps.

(1a) $f(U)$ does not cross $U$ because $f(U) \supset U$.

(1b) $f(U) \cap S=\varnothing$ : This follows because there are no homoclinic points.

(1c) $f(U) \cap C=\varnothing$ : Since $z^{\prime \prime}$, which is the end of $U$, is the closest point of $T^{u} \cap J$ to $S$ among those which $N^{u}(z)=0, f(U)$ could only intersect $C$ by going around $q$. However, $S$ blocks $f(U)$ from returning above $p$ to $J$, and $L^{s}$ blocks it from returning below and around $p$.

(2a) $f(C) \cap U=\varnothing: f^{-1}(U) \subset U$ and $U \cap C=\varnothing$ so $f^{-1}(U) \cap C=\varnothing$.

(2b) $f(C) \cap S=\varnothing: C$ is above $S$ along $J$ (under the current hypothesis). Also, $f^{-1}(S) \cap J$ is below $z^{\prime}$, the end of $S$, because (i) $z^{\prime}$ is the highest point of points in $J \cap R^{s}$ with $N(z)=0$; (ii) $f^{-1}(J)$ is to the left of $J$; (iii) $R^{s}$ can only return by 
coming around $q$; and (iv) $R^{s}$ cannot come around $p$ because it is blocked by $L^{s}$. Therefore $f^{-1}(S) \cap C=\varnothing$.

(2c) $f(C) \cap C=\varnothing$ : If not, then there exists $q \in f(C) \cap C$ with $z \neq f^{-1}(z)$. Thus $\delta_{x}\left(f^{-1}(z)\right)=0$. But the slope of $\left\{\delta_{x}=0\right\}$ is positive so this is impossible.

(3a) $f(S)$ does not cross $\Gamma$ because $f(S) \subset S$.

We have shown that $f(\Gamma)$ does not cross $\Gamma$. Because $U$ is outside $S$ along $J$, $f(D)>D$. This contradicts the area decreasing assumption of $f$. This contradiction shows that $T^{u}$ cannot go outside $R^{s}$ along $J$ and so $R^{s}$ goes outside $T^{u}$.

Because $R^{s}$ cannot cross $T^{u}$ or $L^{s}$, it must remain in the region where $\delta_{x}^{-}<0$. Under further backward iteration, it must leave the left end of $B$ because it cannot go to any fixed point and cannot cross $f^{-1}\left\{\left(x, y_{0}\right): x \geq x_{0}\right\}$. This completes the proof of part (b) of Lemma 3.2.

PROOF OF LEMMA 3.2(c). The part of $R^{s} \cap\left[B \cup f^{-1}(B)\right]$ lies in a compact part by the proof of part (b). Take $S_{1}$ to the part from $p$ to the lowest point of intersection of $R^{s} \cap\left\{x=x_{1}\right\}$, where $\left\{x=x_{1}\right\}$ is the left end of $B$. Similarly, take $S_{2}$ to be the part from $p$ to the highest point of $L^{s} \cap\left\{x=x_{1}\right\}$. Let $C$ be the connecting segment along $\left\{x=x_{1}\right\}$. Let $\left\{\Gamma^{\prime}=S_{1} \cup S_{2} \cup\{p\} \cup C\right.$. Then $\Gamma^{\prime}$ surrounds a region $D_{1}$. An easy argument (a little like the proof of part (b)) shows $f\left(D_{1}\right) \subset D_{1}$. See [11] for details.

\section{REFERENCES}

1. K. Alligood, L. Tedeschini-Lalli and J. Yorke, Metamorphoses: sudden jumps in basin boundaries, University of Maryland, preprint, 1985.

2. K. Alligood and J. Yorke, Accessible saddles on fractal basin boundaries, University of Maryland, preprint, 1987.

3. G. Davis, Homoclinic Tangencies and infinitely many sinks, Thesis, Northwestern University, 1987.

4. R. Devaney and Z. Nitecki, Shift automorphisms in the Hénon mapping, Comm. Math. Phys. 67 (1979), 137-146.

5. R. Devaney, Homoclinic bifurcations and the area-conserving Hénon mapping, J. Differential Equations 51 (1984), 254-266.

6. N. K. Gavrilov and L. P. Silnikov, On three-dimensional dynamical systems close to systems with a structurally homoclinic curve. II, Math. USSR Sb. 19 (1973), 139-156.

7. C. Grebogi, E. Ott and J. Yorke, Metamorphoses of basin boundaries, University of Maryland, preprint.

8. S. Hamel and C. Jones, Jumping stable manifolds for dissipation maps of the plane, preprint, 1986; Ergodic Theory and Dynamical Systems (submitted).

9. S. Newhouse, The abundance of wild hyperbolic sets, Publ. Math. Inst. Hautes Etudes Sci. 50 (1979), 101-151.

10. __ Lectures on dynamical systems, Progress in Math., Birkhäuser, 1980, pp. 1-114.

11. J. Palis and W. de Milo, Geometric theory of dynamical systems, Springer-Verlag, New York and Heidelberg, 1982.

12. S. Patterson and C. Robinson, Basin of sinks near homoclinic tangencies, preprint, Northwestern University, 1986.

13. C. Robinson, Cascade of sinks, Trans. Amer. Math. Soc. 288 (1985), 841-849.

Department of Mathematics, St. John's University, Collegeville, Minnesota 56321

DEPARTMENT OF MATHEMATICS, NORThWESTERN UNIVERSITy, EVANSTON, ILliNOIS 60201 (Current addresss of Clark Robinson)

Current address (Sam Patterson): Department of Mathematics, Carleton College, Northfield, Minnesota 55057 